\title{
Increased Prevalence of Severe Rheumatic Heart Disease Requiring Early Valve Replacement Surgery and Fragmented Germ Cells
}

\author{
Elizabeth Jeya Vardhini Samuel1, Ramesh ${ }^{1}$, K. B. Latha1, K. V. Lalitha², Peter Prashanth², \\ Bosco Emmanuel ${ }^{*}$ \\ ${ }^{1}$ Department of General Medicine, Pondicherry Institute of Medical Sciences, Pondicherry, India \\ ${ }^{2}$ Department of Pediatrics, Pondicherry Institute of Medical Sciences, Pondicherry, India \\ ${ }^{3}$ Central Electrochemical Research Institute (CECRI), Karaikudi, India \\ Email: *elizabethjsamuel@gmail.com
}

Received 24 October 2014; revised 23 November 2014; accepted 3 December 2014

Copyright (C) 2014 by authors and Scientific Research Publishing Inc.

This work is licensed under the Creative Commons Attribution International License (CC BY). http://creativecommons.org/licenses/by/4.0/

(c) (7) Open Access

\section{Abstract}

Background: Patients requiring valve replacement at a younger age, for rheumatic heart disease was apparently increasing after the new age practice of contraception. Methods: In 2012, data of patients referred to tertiary care hospital from two cardiology screening camps conducted in the community, were analyzed for the severity of presentation of rheumatic heart disease and associated contraception status by dividing into the three age groups -20 - 35 years, 36 - 50 years, $>51$ years; 400 patients attended the camps; 31 female and 2 male patients were referred to the tertiary care hospital for further evaluation and categorized treatment, including valve replacement surgeries; among the 33 referred patients, belonging to the three age groups, the data were analyzed for [if any] correlation of contraception status with severe rheumatic heart disease. 8 randomly chosen males, of the 3 age groups, from a different community, whose life partners had undergone sterilization, were assessed for their serum testosterone levels. Results: Among 20 - 35 years, 7 female patients with contraception required valve replacement surgeries [p < 0.0005]; among 36 - 50 years, 20 female patients required valve replacement surgeries [p < 0.0005]; whereas among $>51$ years, 3 female and 2 male patients without contraception received medical treatment only [p < 0.0005]. Conclusion: Concept is contraception with smashed fragmentation of germ cells, consequent reduced endogenous estrogen and androgen leading to defaulted genomic repertoire, deranged cell metabolism and increased tendency for fibrosis has resulted in increased prevalence of severe valve disease in younger age itself, requiring valve replacement.

\footnotetext{
${ }^{*}$ Corresponding author.
}

How to cite this paper: Samuel, E.J.V., Ramesh, Latha, K.B., Lalitha, K.V., Prashanth, P. and Emmanuel, B. (2014) Increased Prevalence of Severe Rheumatic Heart Disease Requiring Early Valve Replacement Surgery and Fragmented Germ Cells. International Journal of Clinical Medicine, 5, 1392-1399. http://dx.doi.org/10.4236/ijcm.2014.521177 


\section{Keywords}

\section{Fragmented Germ Cells, Reduced Endogenous Androgen, Estrogen, Deranged Cell Metabolism, Defaulted Genomic Repertoire, Enhanced Fibrosis}

\section{Introduction}

Rheumatic etiology is a smoldering pathology because it takes about 13 - 15 years before valvular lesions can be identified and it takes 25 years for critical stenosis to develop, requiring valve surgeries; hence we advocate a minimum of 25 years of penidure-benzathine penicillin prophylaxis after identification of rheumatic fever, to thwart valve disease and of course life time prophylaxis after valve lesions are established, to prevent progression of damage in each valve and to prevent progression of damage to involve all the four valves.

In 2008 when a wife underwent tubal recanalisation, adhesion release, for her intractable abdominal pain, the germ cells destruction by contraception had stopped; her endogenous estrogen had returned to $79.9 \%$ of her age related normal levels; her symptoms of abdominal pain had disappeared and her husband who had undergone closed mitral valvotomy $~ 10$ years back, was repeatedly getting admitted prior, requiring oxygen administration; he also improved remarkably after the wife's recanalisation and is only on benzathine penicillin prophylaxis, for the past 6 years, suggesting restoration of genomic repertoire with reversal of contraception by tubal recanalisation, stopping germ cells destruction, restoring endogenous androgen, estrogen in the life partners, restoring the cell metabolism of new cell formation, healing, reduced cicatrisation.

Whereas increased prevalence of severe rheumatic valve diseases, requiring surgeries at younger age e.g. 24 - 34 years old in individuals with tubectomy was increasingly apparent, unlike in the era before contraception [early 20th century] as contraception implementation was steadily achieved. Hence a retrospective data analysis to evaluate altruistic association [if any] of contraception, with quick progression of valvular rheumatic heart disease was planned; decrease in endogenous estrogen after contraception had been documented; [1] among contraception users, endogenous androgen estimation in male life partners, was also attempted, to confirm the concept of androgen reduction, secondary to destruction of germ cells by contraception.

\section{Methods}

In 2012, 400 patients had attended two cardiology camps, conducted 60 days apart in the community; of them 33 patients, including two males, were referred to the tertiary care hospital, for further evaluation and management; their data is analyzed for the association of contraception status and severity of the rheumatic valve pathologies requiring surgery; the patients were divided into the 3 age groups of 20 - 35 years, 36 - 50 years, >51years.

Data of testosterone levels of randomly chosen 8 males, from another community, divided into the 3 age groups, whose life partners had undergone sterilization, was also analyzed for its significance with contraception.

\section{Results}

Among contraceptive users aged 20 - 35 years, 7 fold increased requirement of valve replacement surgeries was observed [p $<0.0005]$, i.e. all the 7 female patients who underwent valve replacement, had undergone permanent sterilization; among contraceptive users aged 36 - 50 years, 20 fold increased requirement of valve replacement surgeries was observed [p < 0.0005], i.e. all the 20 female patients who underwent valve replacement, valvotomy had undergone sterilization; whereas among $>51$ - 70 years 3 female and 2 male patients without contraception, received medical treatment only $[p<0.0005]$. Glaring, obvious difference among contraception and non-contraception users (Figure 1); there was no significant difference by gender.

Plasma testosterone was reduced in males observing contraception, more than $50 \%$ below normal range, for that particular age group e.g. $1.3 \mathrm{ng} / \mathrm{ml}, 1.5 \mathrm{ng} / \mathrm{ml}, 1.8 \mathrm{ng} / \mathrm{ml}$; on analysis among 20 - 35 years of contraception users $66.6 \%$ had significantly reduced levels [p < 0.0005] of testosterone; among contraception users, aged 36 50 years and $>51$ - 70 years, $100 \%$ of males showed gross reduction of testosterone $[\mathrm{p}<0.0005]$ (Figure 2). 


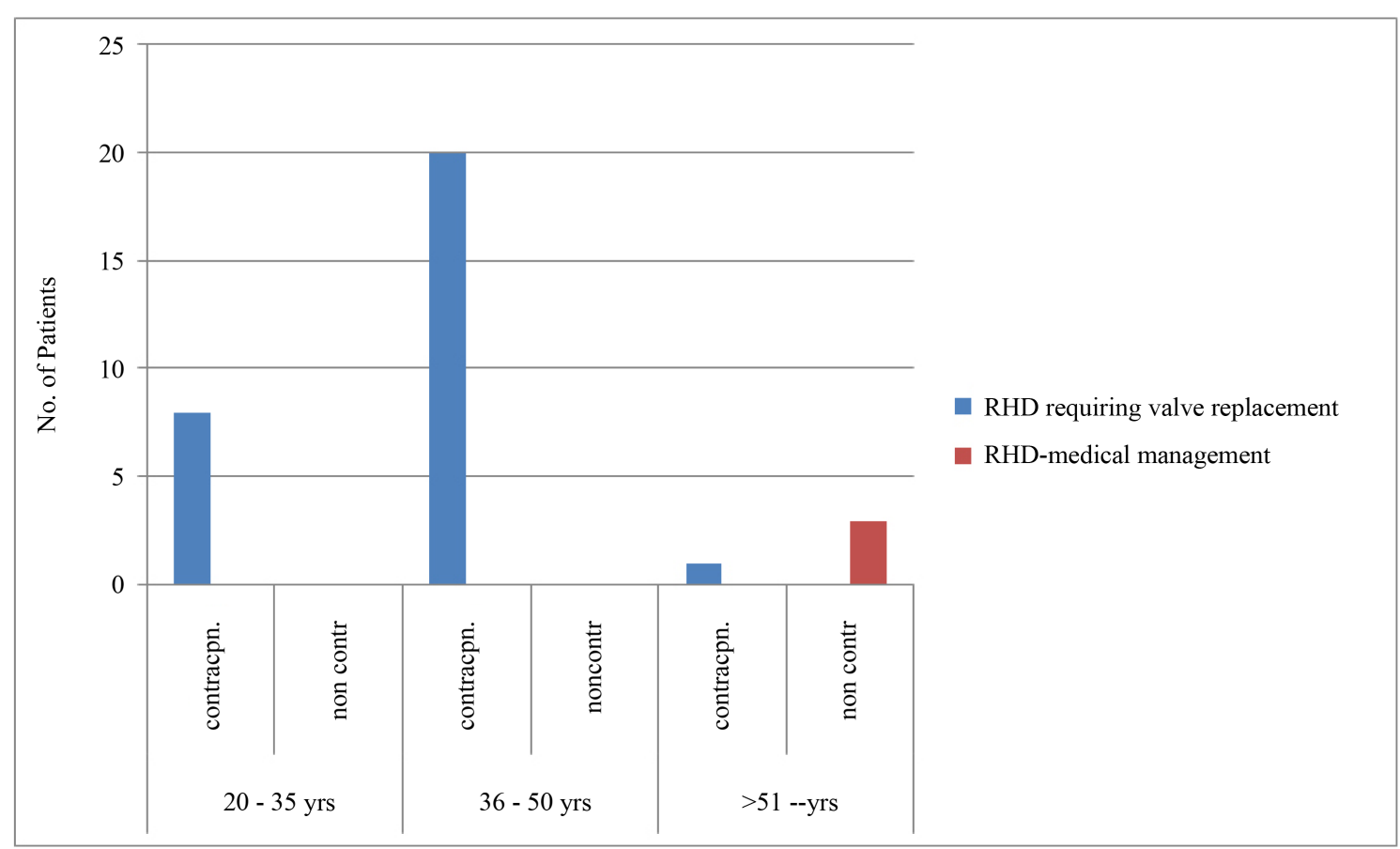

Figure 1. Prevalence of severe rheumatic heart disease and contraception.

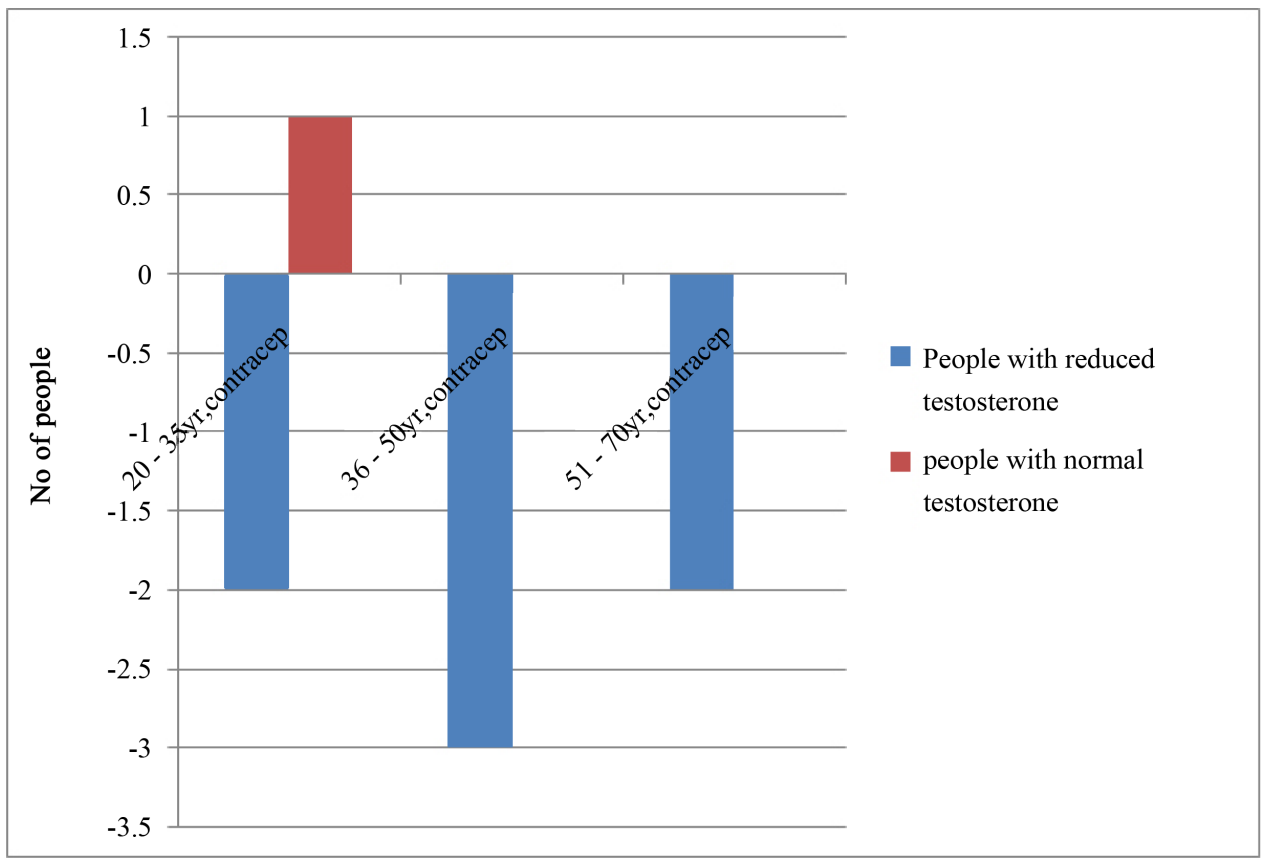

Figure 2. Plasma testosterone levels among male contraception users.

\section{Discussion}

Acute rheumatic fever is a multisystem disease, resulting from auto immune reaction to infection with Group A Streptococcus. All the manifestations resolve except cardiac valvular damage [rheumatic heart disease] [2].

Acute rheumatic fever and rheumatic heart disease are diseases of poverty; were common in all countries until early 20th century; then the incidence declined in industrialized nations, attributable to improved living conditions with less crowded housing, better hygiene, thereby reduced transmission of Group A streptococci; intro- 
duction of antibiotics and improved medical care also had its supplemental effect.

After 1980 recurrent outbreaks of rheumatic fever began in rocky mountain states of the United States where increased rates still persist; in developing countries rheumatic heart disease is common among children and forms a major cause of morbidity and mortality in adults also; 15 - 19 million people in the world have rheumatic heart disease; $1 / 4$ million deaths are attributed to rheumatic heart disease; of these $95 \%$ occur in developing countries. Hot spots with particularly elevated rates include Australasia, Sub-Saharan Africa, Pacific nations and India; coordinated, register based rheumatic heart disease programs will be cost effective.

Acute rheumatic fever is mainly a disease of children of 5 - 14 years, less common in older adolescents and adults; rare in > 30 years; whereas rheumatic heart disease peaks between 25 - 40 years i.e. smoldering pathology spans over $\sim 25$ years before structural valve disease manifests, implying chemoprophylaxis with benzathine penicillin for minimum 25 years is required to prevent the disease manifestation and after structural pathology of valves, life time chemoprophylaxis is required to prevent progression of the disease in severity and involving other valves. Rheumatic heart disease is twice more common in females than males.

Acute rheumatic fever is exclusively caused by upper respiratory tract infection by Group A streptococci; 3\% - 6\% of any population is susceptible; HLA class II alleles is strongly associated with susceptibility; high levels of mannose binding lectin, polymorphisms of transforming growth factor B1 gene, immunoglobulin genes, high level expression of a particular alloantigen present on B cells D8-17 are found in patients with rheumatic fever.

When a susceptible host encounters Group A streptococcal infection, auto immune reaction leads to damage to human tissues by cross reactivity between epitopes on the organism and the host; cross reactive epitopes are present in the streptococcal M protein and the $\mathrm{N}$ acetyl glucosamine of Group A streptococcal CHO which are immunologically similar to molecule in myosin, tryptomysin, keratin, actin, laminin, vimentin; cross reactive antibodies attach to cardiac valve, endothelium, allowing the entry of primed CD4+ T cells, leading to subsequent $\mathrm{T}$ cell mediated inflammation; indolent carditis follow prolonged latent periods; in many patients the Group A streptococcal infection is subclinical requiring antibody testing.

Endocardium, myocardium, pericardium are involved in the pathology; mitral valve is commonly affected; valve disease may remain asymptomatic for many years; main pathology is progressive fibrosis, fusion of valve commissures, shortening of chordae tendinae [3] producing rigidity, distortion of the cusps leading to stenosis.

The concept is contraception results in smashed fragmentation of 20 million germ cells/day to form ring chromosomes, fragmented chromatids [4], acentric fragments, chromatid breaks, which are recognized as foreign leading to autoimmune [5] antibodies and the destructive multisystem diseases, including rheumatic heart disease, triggered by Group A streptococci epitopal cross reactivity.

Concept is destruction of germ cells by contraception is associated with decrease in endogenous estrogen, androgen in partners, with consequent deranged cell metabolism [6] of impaired cell differentiation, growth, increased degeneration, increased apoptosis of cells, reduced new cell formation leading to enhanced fibrosis in tissues including cardiac valves; defaulted genomic [6] repertoire i.e. impairment of embryo like healing in the tissues due to reduced endogenous estrogen, androgen surveillance secondary to contraception, aggravates destructive diseases including quick progression of rheumatic heart disease, enhanced cicatrisation requiring valve replacement surgeries at younger age.

Deranged cell metabolism by contraception includes mutation, acquired polymorphism in transforming growth factor B1 gene, immunoglobulin genes increasing the susceptibility of the host.

Our retrospective data analysis reveals obvious seven fold increase in valve replacement surgeries among contraception users aged 20 - 35 years [p $<0.0005$ ], twenty fold increase in valve surgeries among contraception users aged 36 - 50 years $[p<0.0005]$; whereas $>51$ - 70 years non-contraception users exhibited less severe disease requiring medical treatment.

Estimation of testosterone in male partners among contraception users, showed significantly reduced values in $66.6 \%$ of 20 - 35 years age group [p $<0.0005$ ], $100 \%$ of 36 - 50 years and $>51-70$ years. This reduced androgen, estrogen [1] surveillance results in metabolic syndrome occurring in both partners, secondary to contraception, with its smashed fragmentation of germ cells. Estrogen [1] estimation done in 105 people including both contraception, non-contraception users, among the three age groups of 20 - 35 years, 36 - 50 years and $>51$ years revealed reduced estrogen levels below normal range in $61 \%$ [p $<0.0005]$ among the 3 age groups of contraception users only.

In 1998 Professor Paul Devroey [7] discovered estrogen like particles are on the rise in the air when he attempted to find the air pollutant to explain increase in infertility. 
In 1994 Dr Susan Job ling [8] of Brunel University documented estrogen like compounds in river water as pollutant when she had attempted to elucidate the pollutant responsible for disappearance of fish

$863,000,000$ abortions $\times 4200 \mathrm{pg}$ of estrogen $\times \sim 350 \mathrm{ml}$ blood loss/abortion $=$ environmental estrogen.

When a mother is blessed for e.g. with 10 children she will not menstruate for 200 months or 20 years; whereas after contraception:

$1,989,375,754$ women of $15-50$ years $\times 200$ months $\times 300$ pg of estrogen $\times 350 \mathrm{ml}$ menstrual blood loss $=$ environmental estrogen.

Global environmental estrogen increase = global innocent aborted blood, contraceptive menstrual blood contained air-inhalation, water ingestion = environmental aborted blood, contracepted menstrual blood contained pollution.

Our analysis of sea, river waters, detected estrogen [9], alpha feto protein and $\beta$ human chorionic gonadotropins, further confirming aborted, contraceptive menstrual blood pollution of the environment.

Contraception, abortion with its aborted blood, contraceptive menstrual blood pollution [10] [documented by rise in environmental estrogen, detected alpha feto protein, $\beta$ human chorionic gonadotropins in sea, river water] favors microbial growth, virulence and transmission including Group "A" Streptococcus to explain the return of rheumatic fever in rocky mountain states from 1980, reflecting on the successful global implementation of contraception from mid 20th century.

Contraception reversal restores genomic repertoire in every cell [11] as cause and effect phenomenon (Figure S1) including delay in progression of damage to cardiac valves enhanced healing in the structural valve lesions and improvement in cardiac functional status.

Congenital heart diseases coming for cardiothoracic surgery have decreased drastically because the congenital anomalies are terminated before birth probably; recollecting in 1983, there was a colleague of mine who was doing masters in pathology, had undergone surgical repair for tetralogy of Fallot; he could climb 4th flight of stairs with ease when I would have completed climbing 2nd flight of stairs; such is the technology offered by cardiothoracic surgery and the healing in the cells; ventricular septal defect is known for its spontaneous closure before 7 years of age; we've treated arrested hydrocephalus aged 17 years for lupus nephritis working as driver; how a gene will behave we'll never know; remarkable healing capacity of cells-genomic repertoire; if it's a lethal mutation it'll follow natural demise in spite of our efforts; our field of Life sciences can improve by attempting to find solutions to heal, help them after birth, never by terminating; let not the child be butchered in uteri for congenital anomalies; let the mother at least be informed of the high risk of breast cancer, associated with termination of pregnancy; rather let the parent be counseled about the noble cause of bringing up a differentially abled child, life-a double crown awaits for them in pearly gates of heaven; all the while counseling about preventing congenital anomalies by avoiding unnecessary medications during the first trimester including pills used to terminate, prevent life.

\section{Conclusions}

Contraception, abortion fragments $>20$ million germ cells/day, and consequently defaulted genomic repertoire i.e. embryo like healing in tissues, result in enhanced fibrosis, cicatrisation of fine textured remarkable cardiac valves, leading to quick progression of rheumatic valvular heart disease.

Fragmented germ cells by contraception, abortion being recognized as foreign, result in increased auto immune reaction, increased epitopal cross reactivity with Group "A" streptococcal antigens.

Deranged cell metabolism secondary to contraception results in, acquired polymorphisms of transforming growth factor B1 gene, immunoglobulin genes, increasing the susceptibility of host for rheumatic fever and heart disease.

Hence among contraception users aged 20 - 35 years, 7-fold increased requirement of valve replacement surgeries $[\mathrm{p}<0.0005]$ was noticed, i.e., when they presented first time itself, the disease had progressed to severe valve pathology, requiring valve replacement.

Among contraception users aged $>36$ - 50 years, 20 -fold increased requirement of valve replacement surgeries [p $<0.0005$ ] was noticed, whereas $>51-70$ years non-contraception users required only medical treatment.

Androgen estimation revealed reduction of testosterone in $66.6 \%$ [p $<0.0005]$ of male partners observing contraception among 20 - 35 years and 100\% males among 36 - 50 years and $>51$ years of contraception users showed reduced testosterone levels; this was similar to the $61 \%$ of contraception users with reduced estrogen 
levels in earlier studies.

Contraception reversal, with eradication of global contraception, abortion will reduce the severity, quick progression of rheumatic heart disease by restoring genomic repertoire, regaining robust cell metabolism and reducing aborted, contraceptive menstrual blood pollution, which is a good media for growth, virulence, spread of organisms including Group “A” Streptococcus.

Since rheumatic heart disease is exclusively associated with upper respiratory tract infections with Group "A" Streptococcus, complete treatment with antibiotics to eradicate residual infections also should be prioritized as a measure of prevention. Rheumatic heart disease has smoldering pathology over $\sim 25$ years, often unrecognized, hence chemoprophylaxis for minimum 25 years with benzathine penicillin will prevent manifestation of rheumatic heart disease; once valve disease manifests, life time chemoprophylaxis can prevent progression of the disease in severity spreading to involve the other four valves.

Essential fatty acids rich diet with provision of steroid hormones synthesis (androgen, estrogen) will enable genomic repertoire to protect from degenerative, (50\%) infectious diseases, including rheumatic heart disease.

Reversal of contraception delays progression of the disease and enhances healing of structural valve lesions with benzathine prophylaxis, as a cause and effect phenomenon by restoration of germ cells, endogenous androgen, estrogen, genomic repertoire and regularizes cell metabolism.

\section{Key Points}

- Requirement of valve surgeries in rheumatic heart disease, increase by seven fold among contraception users aged $>20$ - 35 years and twenty fold among contraception users aged $>36$ - 50 years.

- Chemoprophylaxis with benzathine penicillin for 25 years can prevent manifestation of rheumatic heart disease; after disease manifestation life time chemoprophylaxis prevents progression of rheumatic heart disease.

- Antibiotics to eradicate respiratory tract infections without residue will prevent rheumatic fever.

- Contraception reversal, eradication of global contraception, abortion will reduce the prevalence, severity of rheumatic heart disease.

- Aborted blood, contraceptive menstrual blood pollution favors microbial growth, including Group "A" Streptococcus.

- Essential fatty acids rich diet provides normal endogenous estrogen, androgen synthesis with excellent genomic repertoire.

- Endogenous androgen is reduced in $66.6 \%-100 \%$ of male partners of contraception users secondary to fragmentation of germ cells.

\section{Acknowledgements}

We acknowledge with thanks, Department of Electronics Data Processing [EDP] unit of Pondicherry Institute of Medical Sciences [PIMS] who enabled us with figures.

\section{Conflicts of Interests}

None declared.

\section{References}

[1] Elizabeth, J.S., Gouthaman, T. and John, K.T. (2014) Abortion, Contraception Reversal-Medical Miracle-Autologous Germ Cells Replant-Decline in Global Degenerative, Autoimmune, Neoplastic, Infectious Diseases; Decline in Rising Environmental Estrogen [Equating with Aborted Blood Pollution] and Decline in Depletion of Ozone. IOSR Journal of Dental and Medical Sciences, 13, 93-97. http://dx.doi.org/10.9790/0853-139193102

[2] Carapetis, J.R. (2012) Rheumatic Fever; Harrison's Principles of Internal Medicine. 18th Edition, Vol. 2, $2752-2753$.

[3] Boon, N.A., Fox, K.A.A., Bloomfield, P. and Bradbury, A. (2002) Cardiovascular Disease; Davidson’s Principles and Practice of Medicine. 19th Edition, 453.

[4] Gilliland, B.G. (1987) Systemic Sclerosis; Harrison’s Principles of Internal Medicine. 11th Edition, Vol. 2, 1429.

[5] Elizabeth, J.S., Gouthaman, T., George, S.J. and John, K.T.K. (2014) Autoimmunity and Fragmented Germ Cells. IOSR Journal of Dental and Medical Sciences, 13, 99-102. http://dx.doi.org/10.9790/0853-139699105

[6] Mendelsohn, J. (1987) Principles of Neoplasia; Harrison’s Principles of Internal Medicine. 11th Edition, 422-423. 
[7] Devroey, P. (1998) Rise in Estrogen Like Particles in the Air; Fertile Ground. Odyssey Journal, 4, 4.

[8] Jobling, S. (1996) A Survey of Estrogenic Activity in United Kingdom Inland Waters. Environmental Toxicology and Chemistry, 15, 1993-2002. http://dx.doi.org/10.1002/etc.5620151118

[9] Elizabeth, J.S., Sanjoy, J.G., Gouthaman, T. and Jobin, J. (2014) Increased Prevalence of Liver Diseases and Fragmented Germ Cells with Reduced Endogenous Estrogen; Increasing Prevalence of Hepatitis B Virus and Rising Environmental Estrogen. International Journal of Scientific and Engineering Research, 5, 776-777.

[10] Elizabeth, J.S., Gouthaman, T., George, S.J., Tony, J., Peter, D., John, J. and David, B. (2014) Increased Prevalence of Dermographism, Filariasis, Mosquito Borne Diseases and Rising Environmental Estrogen Equating with Aborted Blood, Contraceptive Menstrual Blood Pollution. International Journal of Scientific Research and Engineering Studies, 1, 5-8.

[11] Elizabeth, J.S., Gouthaman, T. and Kevin, T.J.K. (2014) Abortion, Contraception Reversal-Medical Miracle-Autologous Germ Cells Replant-Decline in Global Degenerative, Autoimmune, Neoplastic, Infectious Diseases; Decline in Rising Environmental Estrogen [Equating with Aborted Blood Pollution] and Decline in Depletion of Ozone. IOSR Journal of Dental and Medical Sciences, 13, 93-102. http://dx.doi.org/10.9790/0853-139193102

[12] Elizabeth, J.S. (2014) Increased Prevalence of Hernia, Uterine Descent, Fibrous Adhesions, Retroperitoneal Fibrosis and Germ Cells with Endogenous Estrogen. International Journal of Innovative Research and Studies, 3, 187-194. 


\section{Supplementary}
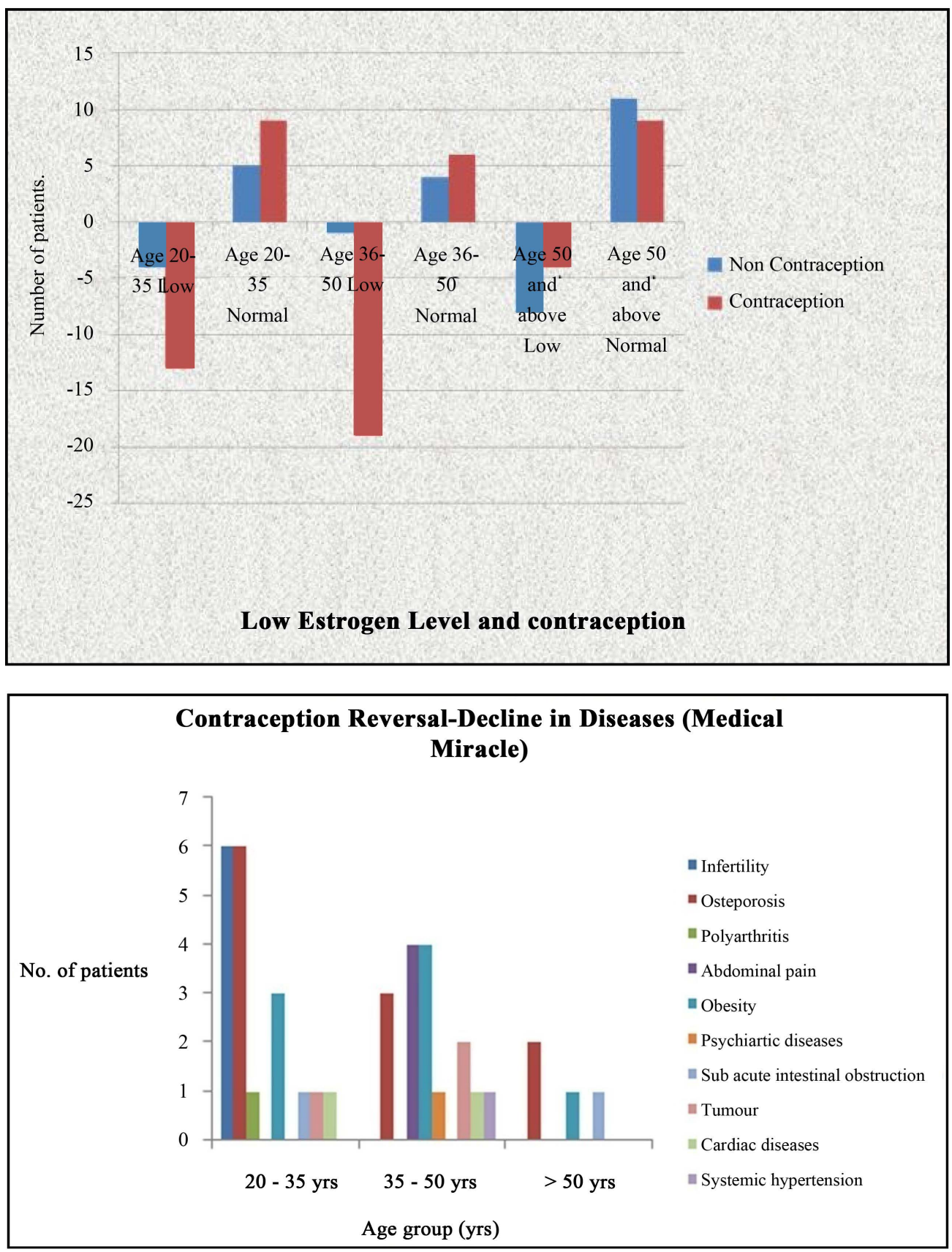

Figure S1. Regression of rheumatic heart disease with contraception reversal [12]—cause and effect phenomenon. 
Scientific Research Publishing (SCIRP) is one of the largest Open Access journal publishers. It is currently publishing more than 200 open access, online, peer-reviewed journals covering a wide range of academic disciplines. SCIRP serves the worldwide academic communities and contributes to the progress and application of science with its publication.

Other selected journals from SCIRP are listed as below. Submit your manuscript to us via either submit@scirp.org or Online Submission Portal.
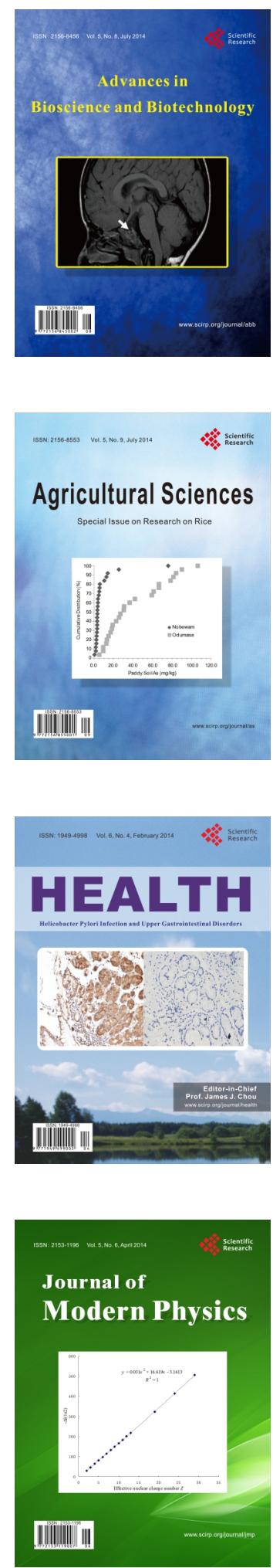
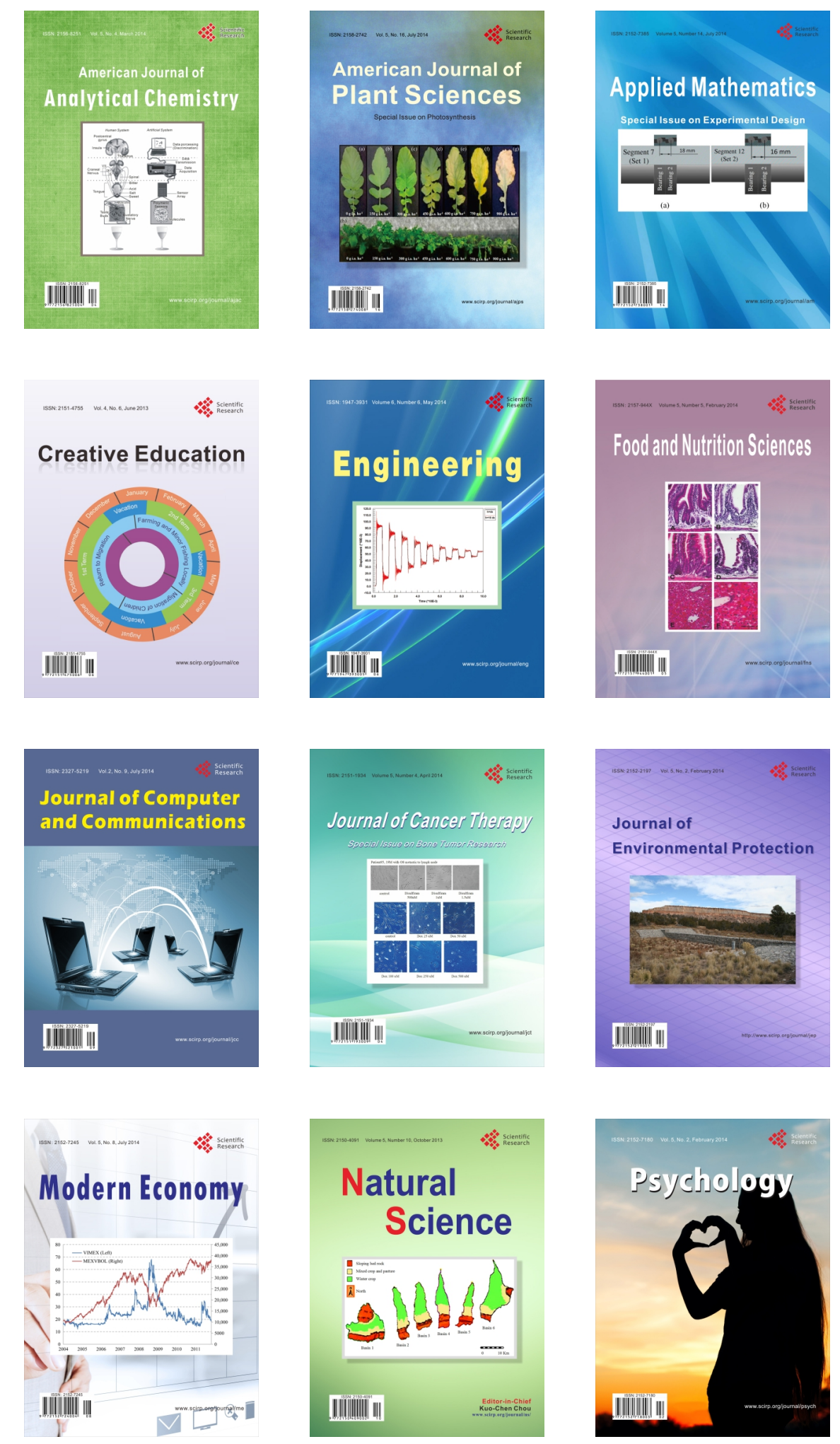